\title{
Neoatherosclerosis: Inevitable Destination or Next Target?
}

\author{
Taishi Yonetsu, MD and Tsunekazu Kakuta, MD, PhD* \\ Division of Cardiovascular Medicine, Tsuchiura Kyodo General Hospital, Ibaraki, Japan
}

\begin{abstract}
Progression of atheromatous plaque within neointima of coronary stents, which was termed neoatherosclerosis, has gained interest as a potential cause of very late stent failure. Pathologically, neoatherosclerosis was defined as the development of foamy macrophage clusters, fibroatheroma, thin-cap fibroatheroma, plaque rupture, and in-stent calcification within the stent. Following the pathological observations, a growing number of in vivo studies by using optical coherence tomography (OCT) and coronary angioscopy have been published to date. Those studies tried to clarify the features of neoatherosclerosis and have improved our knowledge substantially. Nevertheless, there still are missing information on natural history and clinical implications of neoatherosclerosis. The present review article summarized the definitions of neoatherosclerosis from the stands of view of pathology, OCT, and angioscopy. Moreover, what is known and what is not known regarding neoatherosclerosis were outlined for better understanding of pathophysiological consequences of coronary stents, which hopefully generates further ideas of clinical investigations.
\end{abstract}

Key words: stent thrombosis, restenosis, optical coherence tomography, coronary angioscopy

\section{Introduction}

Since the balloon coronary angioplasty was introduced in 1970s, percutaneous coronary intervention (PCI) has gained widespread acceptance as the primary mode of treatment for coronary artery disease. ${ }^{1)}$ Although balloon angioplasty gives mechanical dilatation of narrowed coronary arteries, it bares potential risks for acute lesion closure within a day after the procedure, which is associated with vessel recoiling. Metallic stents were invented to compensate the recoiling of stretched vessel. ${ }^{2)}$ Stents sufficiently reduced a risk of acute closure after PCI, however, there remained a problem named in-stent restenosis characterized by an excessive neointimal hyperplasia inside the stent, which became the major limitation of coronary stents. In early 2000s, the advent of drug eluting stents (DES) brought a hope to overcome the limitation of stents with its pharmacological suppression of neointimal hyperplasia, and succeeded to improve the restenosis rate after implantation. ${ }^{3)}$ Nevertheless, different difficulties including stent thrombosis (ST) that may result in fatal events have been left to contemporary DES. Obser-

\footnotetext{
* 4-4-1 Otsuno, Tsuchiura, Ibaraki 300-0028, Japan e-mail: kaz@joy.email.ne.jp (Received 2016.06.21; Accepted 2016.11.11)

doi.org/10.15791/angioscopy.re.16.0012
}

vational studies revealed a continuous risk of ST after first-generation DES implantation, which is not completely solved even with the use of newer generation DES. ${ }^{4,5)}$ Thus, the great developments of PCI technologies have been accomplished with the substantial struggle to overcome the limitations of themselves. Based on pathological and clinical studies, ST was attributed to delayed arterial healing, hypersensitive reaction, and newly developed atherosclerosis within the stents after DES implantation. Among these concerns with contemporary DES, new development of atherosclerotic change within the stent, so called neoatherosclerosis, has gained an increasing interest in recent years as a potential cause of very late stent failure after both bare-metal stents (BMS) and DES implantation. The current review article sought to summarize the definition and clinical implication of neoatherosclerosis from the point of views on pathology and intracoronary imagings.

\section{Pathological Definition of Neoatherosclerosis}

Approximately 3 weeks after BMS implantation, stents are endothelialized and begin to be covered by thin neointimas composed of smooth muscle cell. ${ }^{6,7)}$ Excessive growth of neoinitma reduces the lumen area, which consequently leads to restenosis. Angiographic studies reported that typical restenosis of BMS occurs 3-6 months after implantation, and neointimal hyperpla- 
sia shows regression beyond 1 year after implantation. ${ }^{8)}$ Therefore, in the era of BMS, restenosis was believed to be a benign entity that slowly progresses and develops stable angina pectoris. However, some studies suggested that acute coronary syndrome (ACS) is a common presentation of stent failure even after BMS implantation, ${ }^{9-11)}$ which raised a question whether progression of atherosclerotic change within stents can cause plaque rupture and thrombus formation resulting in ACS like native coronary arteries. Nakazawa et al. reported the frequency of pathological atherosclerosis in a total of 406 stented coronary lesions of 299 autopsy cases. ${ }^{12)}$ In their study, atherosclerotic change of neointima was characterized by foamy macrophage clusters, fibroatheromas, thin-capped fibroatheromas, ruptured plaque, and calcified plaque by histopathological assessment within the neointima, and the authors named it "neoatherosclerosis". Neoatherosclrosis was more frequently observed in DES than in BMS. Of note, approximately $30 \%$ of DES showed a atherosclerotic change in stents implanted within 2 years, whereas no BMS exhibited any atherosclerotic changes within the stents. In histology, the earliest finding of neoatherosclerosis is thought to be foamy macrophage clusters that appear around stent struts or beside the lumen. ${ }^{13)}$ The further accumulation of foamy macrophage may induce a formation of fibroatheroma within neointima. Moreover, an advanced fibroatheroma bares necrotic core that generally contains acellular debris with a substantial amount of free cholesterol. Further infiltration of foamy macrophage into neointima makes the fibrous cap of the necrotic core thinner and thin-capped fibroatheroma (TCFA) is formed. Those changes are thought to progress more rapidly in stented segment than in native coronary artery where an atherosclerosis grows taking several decades. Although the definite mechanisms of accelerated development of neoatherosclerosis in stented segments has not been clarified to date, pathologists speculated that incomplete and dysfunctional endothelialization plays a key role, ${ }^{13)}$ which is corroborated by earlier development of neoatherosclerosis in DES than in BMS. In the process of vascular healing after stent deployment, DES induces incomplete maturation of the regenerated endothelium, which is characterized by poor cell-to-cell junctions. Poorly formed cell junctions do not fully function as a barrier to prevent the migration of lipoproteins into sub-endothelial space. Even in BMS, neoatherosclerosis develops within 5-10 years, which is earlier than atherosclerosis of native coronary arteries. Therefore, it is conceivable that metallic material itself may impair the complete endothelialization. Further experimental studies would be warranted to confirm those speculations.

\section{Neoatherosclerosis Identified by Angioscopy}

Although the term of "neoatherosclerosis" was proposed by pathological and optical coherence tomography (OCT) studies, coronary angioscopy had been applied to assess the neointimal coverage and its characteristics by color for more than two decades, in advance to the advent of OCT. ${ }^{14,15)}$ Moreover, in vivo studies using angioscopy had reported the development of a "new" atheromatous plaque within a stent before OCT studies reported neotherosclerosis. ${ }^{16,17)}$ Higo et al. investigated 57 sirolimus eluting stents with serial angioscopic observations at baseline and 10 months after implantation. ${ }^{16)}$ The maximal yellow color grade significantly increased from the baseline to follow-up $(1.4 \pm 1.1$ vs. $1.9 \pm 0.6, \mathrm{p}<0.001)$, which indicated the development of atheromatous neointima after stent implantation. Of interest, yellow grade neointima was associated with thrombus detected by angioscopy, which suggested that a newly developed atheromatous in-stent plaque may be a potential cause of thrombosis. Recently, the DESNOTE study by Ueda et al. has revealed the impact of yellow neointima with the stent on the future risk of very late stent failure (VLSF). ${ }^{18)}$ They enrolled 360 consecutive patients undergoing follow-up angioscopy at 1 year after DES implantation. Patients were stratified by the presence of yellow neointima within the stent by coronary angioscopy, and the incidence of VLSF including cardiac death, myocardial infarction related to the target lesion, and target lesion revascularization were compared. During the follow-up interval of $1,558 \pm 890$ days, VLSF occurred more frequently in patients with yellow neointima than in those without yellow neointima ( $8.1 \%$ vs. $1.6 \%$; log-rank $\mathrm{p}=0.02)$. This study demonstrated the evidence connecting the presence of neoathrosclerosis to clinically significant stent failure, and the furthermore, suggested the need for medical interventions for the high-risk neointima characterized by yellow color in angioscopy. Because of the requirement of investigators' skills for sufficient image quality and limited field of views, the angioscopic investigation for in-stent neoatherosclerosis has not been widely performed so far. Nevertheless, angioscopy enables specific identification of superficial lipid with a relatively simple manner using visual assessment of the color of neointima, which have provided clinically-relevant information on the instability of neointima, ${ }^{19,20)}$ therefore, further studies are warranted for better prediction of stent failure and monitoring the effectiveness of secondary preventions. The information on the comparison of neointimal findings between angioscopy and OCT has been limited. In native coronary lesions, it was reported that the yellow grade plaque by angioscopy inversely correlated with the fibrous cap thickness mea- 
sured by OCT, and best cut-off point to discriminate yellow plaques was $110 \mu \mathrm{m}$ with high sensitivity and specificity. ${ }^{19)}$ However, it is unknown whether the cut-off threshold can be adapted to the assessment of neointima. In fact, a comparison study of OCT and angioscopy exhibited that in-stent yellow segment detected by angioscopy showed various patterns on OCT images, besides lipid-laden neointima. ${ }^{21)}$ Figure 1 shows three different types of OCT findings corresponding to yellow neointima by angioscopy. Further investigation using multiple modalities is worth examining for better understanding of pathophysiology and potentially more precise evaluation of neointima with the combinations of imaging modalities.

\section{Neoatherosclerosis Identified by Optical Coherence Tomography}

Neoatherosclerosis has been investigated in vivo predominantly by OCT with unprecedented resolution and ability of tissue characterization. ${ }^{22,23)}$ OCT is a novel intravascular imaging modality developed in early $2000 s,{ }^{24)}$ which provides higher resolution images in comparison with intravascular ultrasound, with the radial resolution of $10-15 \mu \mathrm{m}^{25)}$ One of the most valu- able advantages of OCT is a capability of plaque characterization. Intracoronary OCT enables identification of the main components of coronary plaques including fibrous plaque, lipid-rich plaque, and calcification. ${ }^{26)}$ On OCT images, fibrous plaque is characterized by high backscattering and a relatively homogeneous signal. Lipid-rich plaque is depicted as a signal-poor region covered by a diffusely bordered, homogeneous signal-rich bands that represents a fibrous cap. Calcification appears as a signal-poor or heterogenous region with a sharp border. Moreover, macrophage accumulation can be visualized by OCT as signal-rich, distinct, or confluent punctate regions that exceed the intensity of background speckles. ${ }^{25,27)}$ Tissue characterization by OCT has been applied to the evaluation of neointimal tissues after stenting. Gonzalo et al. demonstrated various patterns of neointimal tissues such as homogenous, heterogenous, and layered pattern, ${ }^{28)}$ and these information are not available by intravascular ultrasound (IVUS) because of the limited image resolution and poor signal contrast. With use of a superior ability of OCT to visualize neointimal tissue, Takano et al. reported the development of lipid-laden neointima after stent implantation, which is the first OCT study demonstrating neoatherosclerosis
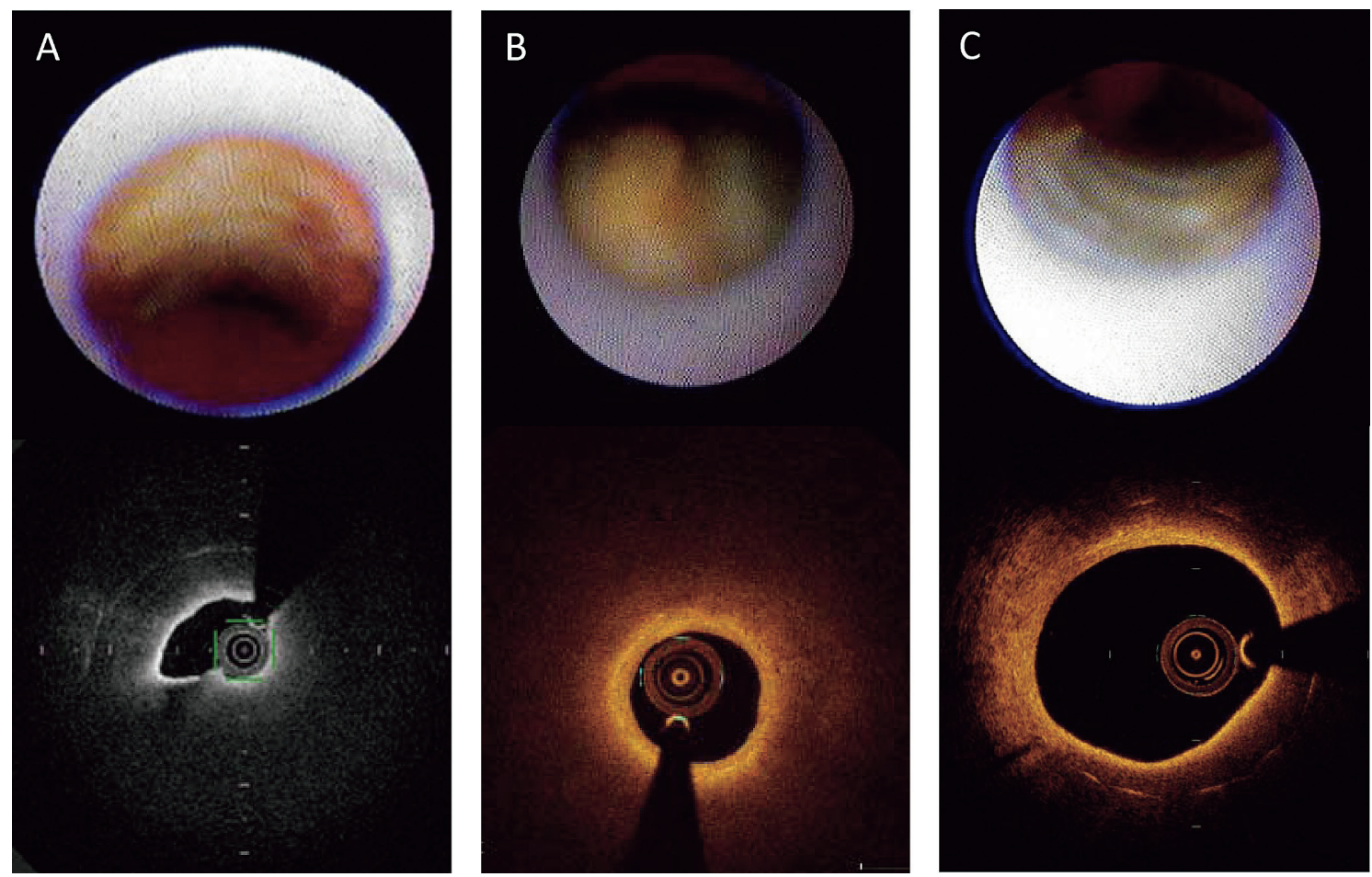

Fig. 1 Yellow neointima by angioscopy and corresponding OCT images

(A) A bare-metal stent at 11 years since implantation in a 71-year-old male. Intensive yellow neointima with red thrombus was observed in angioscopy (upper panel). Optical coherence tomography (OCT) exhibited thin-cap fibroatheroma with a disruption of fibrous cap (lower panel). (B) A sirolimus-eluting stent at 6 years since implantation in a 70-year-old male. Intensive yellow neointima was observed in angioscopy (upper panel). OCT showed circumferential lipid-laden neointima with thick fibrous cap precluding visualization of struts (lower panel). (C) Bare-metal stents at 7 years after implantation in 62-year-old male. A light-yellow neointima was observed in angioscopy (upper panel). OCT exhibited superficial accumulation of macrophage that does not preclude struts visualization. 
although they used a different term. ${ }^{22)}$ Twenty BMS in the early phase ( $<6$ months) and 21 BMS in the late phase ( $>5$ years) after implantation were compared in their study with use of OCT regarding the appearance of neointimal tissue. Lipid-laden neointima was significantly more frequent in late phase BMS as compared with early phase BMS $(67 \%$ vs $0 \%$, p < 0.05). More importantly, BMS in late phase demonstrated intimal disruption and thrombus formation like culprit lesions of ACS in native coronary arteries. Following their studies, numerous OCT studies focused on neoatherosclerosis using different OCT definitions. $^{23,29-31)}$ Those observational studies commonly included lipid-laden neointima and calcification within the stents as the definition of OCT-derived neoatherosclerosis (Fig. 2). However, the inclusion of microstructures such as neovascularization and macrophage accumulations varies among those studies. One of the pitfalls of OCT interpretation is a superficial shadow appearing like a TCFA on OCT images because of signal attenuation behind a bright adluminal layer. These lesions were reported to correspond to dense macrophage accumulations in ex-vivo examinations. ${ }^{32,33)}$ When we consider that both TCFA and superficial accumulation of macrophage are forms of pathological neoatherosclerosis, it might be reasonable to include superficial shadows on OCT into the definition of OCT-derived neoatherosclerosis even if we could not tell whether the findings correspond to TCFA or macrophages. In general, the interpretation of OCT images certainly depends on subjective analysis. Therefore, clear definition that is relevant to clinical data needs to be established in the future studies.

\section{Observational Studies of Neoatherosclerosis}

At almost the same time as the first pathological report focused on "neoatherosclerosis", ${ }^{12)}$ an OCT study by Kang et al. was published demonstrating a significant association between the presence of neoatherosclerosis and clinical presentation in patients with stent failure. ${ }^{23)}$ A total of 50 patients, including 30 stable and 20 unstable angina patients, with in-stent restenosis of DES were investigated. Patients with unstable angina showed a thinner fibrous cap (55 [IQR 42-105] $\mu \mathrm{m}$ vs 110 [IQR 60-205] $\mu \mathrm{m}, \mathrm{p}=0.006$ ), more frequent thin-cap fibroatheroma (TCFA) (75\% vs $37 \%, p=0.008)$, ruptured neointima (75\% vs $47 \%$, $\mathrm{p}=0.044)$, and thrombus ( $80 \%$ vs $43 \%, \mathrm{p}=0.010)$. Multiple OCT studies have reported the correlation between the development of neoatherosclerosis and the time period from BMS implantation. ${ }^{22,34-36)}$ Habara et al. compared OCT findings of neointima in very late restenoses more than 5 years from implantation $(n=43)$ and early restenoses within one year from implantation $(\mathrm{n}=39) .{ }^{35)}$ Stents with very late restenosis showed more heterogenic neointima and disrupted neointima as compared with early restenosis $(90.7 \%$ vs $17.9 \%, \mathrm{p}<0.001$ and $18.6 \%$ vs $0 \%$, $\mathrm{p}<0.03$, respectively). Following the pathological study showing an earlier development of neoatherosclerosis in DES as compared with BMS, a retrospective OCT analysis of MGH OCT registry revealed more frequent lipid-rich neointima in DES as compared with BMS in early ( $<9$ months) and intermediate (948 months) phase (37\% vs. $8 \%, \mathrm{p}=0.02$ and $63 \%$ vs. $28 \%$, $\mathrm{p}=0.03$, respectively), whereas no significant difference was
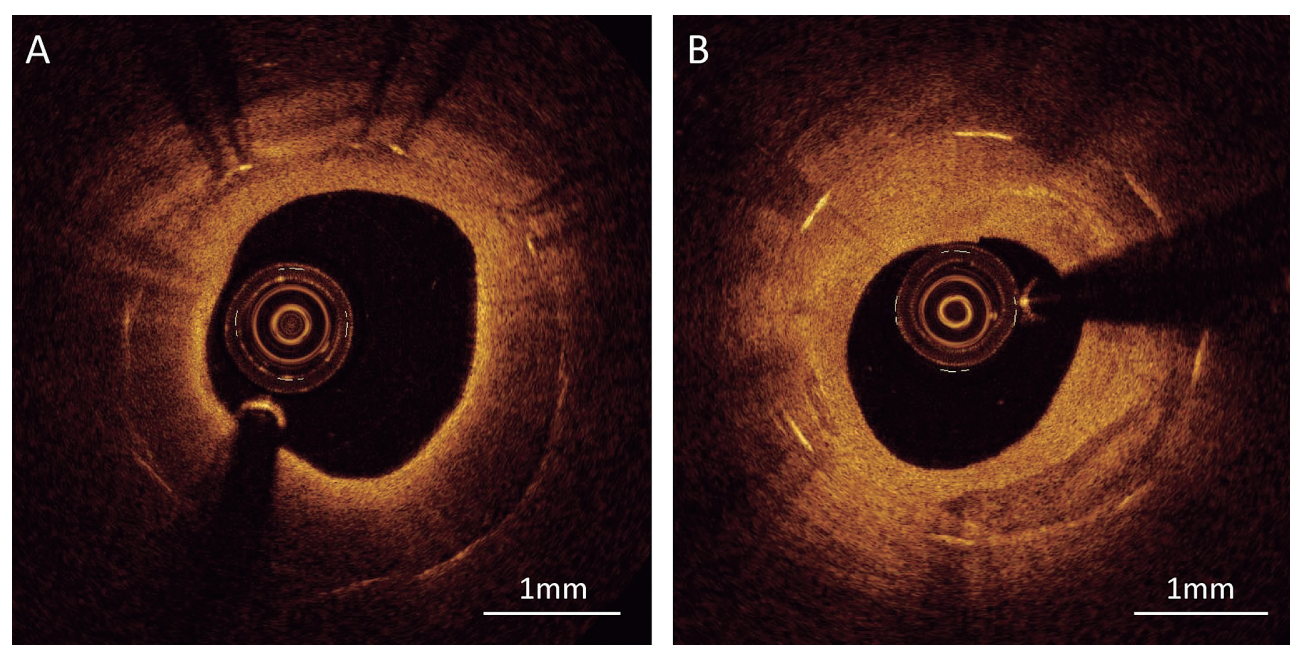

Fig. 2 OCT defined neoatherosclerosis

(A) Lipid laden neointima showing signal poor region with an overlying signal rich band corresponding to fibrous cap. (B) In-stent calcification showing signal poor region with a clear border. 
observed in delayed phase (>48 months) (77\% vs. $75 \%$, $\mathrm{p}=0.99)$ when stents with more than $100 \mu \mathrm{m}$ of neointima were investigated. ${ }^{30)}$ This study corroborated the speculation advocated by pathologists that the time course of neoatherosclerosis development is different between DES and BMS. Thus, multiple OCT studies have consistently reported that DES and longer time duration after stenting are major drivers of neoatherosclerosis. $22,23,30,31,35)$ Other predictors for neoatherosclerosis have been identified by some retrospective analyses using OCT. In an OCT study investigating 179 stents, stent age $>48$ months (OR 4.48 [95\% CI 2.68-9.65]), DES (OR 2.66 [95\% CI 1.38-5.16]), patient's age $>65$ years old (OR 1.91 [95\% CI 1.05-3.44), current smoker (OR 2.30 [95\% CI 1.10-4.82), chronic kidney disease (CKD) (OR 4.17 [95\% CI 1.42-12.32]), and the use of angiotensin converting enzyme inhibitors or angiotensin II receptor blocker (OR 0.42 [95\% CI 0.22-0.80]) were independently associated with the presence of neoatherosclerosis. ${ }^{29)}$ Another retrospective study including $212 \mathrm{DESs}$ from an OCT registry data identified CKD (OR 4.11 [95\% CI 1.09-15.58]), low-density-lipoprotein (LDL)-cholesterol-cholesterol level $>70 \mathrm{mg} / \mathrm{dl}$ (OR 2.53 [95\% CI 1.05-6.08]), and stent age (OR 1.71 [95\% CI 1.40-2.08]) as predictors of neoatherosclrosis. ${ }^{31)}$ Of interest in these studies, ${ }^{29,31)}$ the type of DES was not associated with the frequency of neoatherosclerosis in the multivariate analysis, which were consistent with autopsy studies. ${ }^{37)}$ Although the second-generation DES, such as everolimus eluting stents, zotarolimus eluting stents, and biolimus eluting stents have been shown to compensate some of the limitations of DES including delayed endothelial coverage that is related with the risk of stent thrombosis, ${ }^{37-39)}$ they may not have superior protective effect on the inhibition of neoatherosclerosis as compared to the first-generation DES.

\section{Clinical Implication of Neoatherosclerosis}

Uncovered or malapposed struts have been recognized as hallmarks of late stent thrombosis in pathological and OCT studies. ${ }^{40,41)}$ The advent of OCT, providing detailed in vivo information on neointimal tissues, shed light on neoatherosclerosis as another substrate of late and very late stent thrombosis (VLST). Some OCT studies confirmed that neoatherosclerosis accounts for a certain proportion of VLST. ${ }^{42-44)}$ In the data of the PESTO (Morphological Parameters Explaining Stent Thrombosis assessed by OCT) registry, Souteyrand et al. assessed the OCT findings of 120 stent thrombosis cases including 80 VLSTs. Strut malapposition (34\%), neoathrosclerosis (22\%), stent underexpansion (11\%), evagination (8\%), uncovered struts $(8 \%)$, edge-related disease progression $(8 \%)$, and neointimal hyperplasia (4\%) were identified as potential causes of VLST. ${ }^{43)}$ Tani- waki et al. also studied 64 patients with VLST in a multicenter registry and a total of 58 DES were evaluable by OCT. In their analysis, the leading cause of VLST was strut malapposition $(34.5 \%)$ followed by neoatherosclerosis $(27.6 \%)$, uncovered struts $(12.1 \%)$, and stent underexpansion $(6.9 \%) .{ }^{44)}$ These studies consistently reported that neoatherosclerosis was the second leading cause of VLST following stent malapposition. If stent malapposition is decreased by active use of intravascular images and stent coverage is improved by newer DES technology in the future, neoatherosclerosis would become the major substrate of VLST as a residual flaw left to the contemporary PCI. In addition to the association with VLST, neoatherosclerosis has been reported to be relevant to the progression of remote lesions and future adverse cardiac events. ${ }^{45,46)}$ In an analysis of SIRTAX-LATE OCT cohort, a subgroup cohort of SIRTAX (Sirolimus-Eluting Stent Compared With Paclitaxel-Eluting Stent for Coronary Revascularization) study, in-stent OCT findings at 5 years from implantation and serial changes of angiographic stenosis in non-stented segments during 5 years follow-up were investigated in a total of 88 patients. In their prospective cohort who had received the first-generation DES implantation, OCT assessment at 5 years identified 14 stents with neoatherosclerosis $(15.9 \%)$, which is an important information to know the incidence of neoatheorosclerosis in the real world. Moreover, serial quantitative coronary angiography (QCA) analyses at baseline and 5-year follow-up revealed that the reduction in minimal lumen diameter (MLD) in the non-targeted segments were significantly greater in patients with neoatherosclerosis as compared with those without, which consequently led to more frequent non-target lesion revascularization in patients with neoatherosclerosis. ${ }^{45)}$ Corroborating the data from SIRTAX-LATE OCT study, a retrospective analysis of a single-center OCT registry demonstrated that the presence of neoatherosclerosis was significantly associated with more frequent major adverse cardiac events. A total of 175 patients with 314 stented lesions undergoing follow-up OCT at $>1$ year were selected from the registry data, and the incidence of major adverse cardiac events (MACE) were compared between the patients with neoathrosclerosis in any of stents and those without neoatherosclerosis. MACE including cardiac death, myocardial infarction, clinically-driven target lesion revascularization occurred more frequently in those with neoatherosclerosis than in those without. Those findings may suggest that neoatherosclerosis is a potential marker of high-risk patients for future cardiovascular event. Furthermore, detection of neoatherosclerosis may help monitor the patients during secondary prevention. 


\section{Future Directions}

As described in this article, neoatherosclerosis is acquiring an increasing interest as a residual limitation of drug-eluting stents. Nevertheless, the clinical impact of neoatherosclerosis is still unknown because of the limited information on the natural history of neoatherosclrosis. As most of the previous studies with regard to neoatherosclerosis were retrospective observations, the true incidence, in other words, what percentage of stents develop neoatherosclerosis after follow-up periods has not been sufficiently investigated. Furthermore, we should also explore how often a neoatherosclerosis trigger a stent thrombosis or promote a restenosis, which may contribute to better risk stratification. Although a retrospective study has shown a significant association between the presence of neoatherosclerosis and LDL-cholesterol level, ${ }^{31)}$ there is no prospective study proving the protective effect of statin against the development or progression of neoatherosclerosis. Further investigations are warranted to search an effective medical intervention to prevent adverse events caused by neoatherosclrosis. Moreover, empiric, invasive intervention is not indicated when a neoatherosclerosis is detected within a stent unless the lesion limits the coronary flow. However, if it is possible to identify a type of neoatherosclerosis that causes stent thrombosis with extremely high probability, it might be reasonable to cover or modify the high-risk neoatherosclerosis by PCI with use of drug-coated balloon or bioabsorbable scaffold to prevent subsequent thrombosis.

In order to identify the high-risk features of neoatherosclerosis, further investigations with imaging modalities are essential. To date, coronary angioscopy and OCT are the standard in vivo modalities to evaluate neoatherosclerotic change within the stent. However, a combination or comparison of those two modalities has not been sufficiently tested. As angioscopy and OCT have device-specific advantages and limitations as an intracoronary imaging modality, differential indications of those modalities deserves further consideration. Although yellow neointima may be a specific finding for lipid-laden neointima with thin fibrous cap, one of the major limitation of angioscopy is a limited field of view that may limit the sensitivity. For maintaining its sensitivity, lesion morphology that is suitable for angioscopic investigation should be established.

In addition to intracoronary imaging, non-invasive modality to detect neoatherosclerosis is needed for a broad clinical implication. The presence of neoatherosclerosis can be defined only by histopathology or intracoronary imaging modalities at present, specifically OCT and angioscopy, which require considerably invasive procedures. If a neoatherosclerosis can be non-invasively identified in future, more precise risk stratification for very late stent thrombosis will be available, and moreover, neoatherosclerosis can be a target of secondary prevention. Ultimately, new technologies precluding the development of neoatherosclerosis would be desired, for which bioabsorbable scaffold and polymer may play a part.

\section{Conclusions}

Neoatherosclerosis is considered as one of the remaining issues of the latest coronary intervention, which potentially causes very late stent thrombosis. Although pathological, OCT, and angioscopic studies have revealed the feature of neoatherosclrosis, there as yet is no sufficient evidence connecting the presence of neoatherosclerosis to clinical application. Further studies are required to accumulate the knowledge on clinical implication of neoatherosclerosis.

\section{Disclosure Statement}

Conflict of interest: none declared.

\section{References}

1) Mozaffarian D, Benjamin EJ, Go AS, et al: Heart Disease and Stroke Statistics-2016 Update: A Report From the American Heart Association. Circulation 2016; 133: e38-360

2) Schatz RA, Palmaz JC, Tio FO, et al: Balloon-expandable intracoronary stents in the adult dog. Circulation 1987; 76: 450457

3) Morice MC, Serruys PW, Sousa JE, et al: A randomized comparison of a sirolimus-eluting stent with a standard stent for coronary revascularization. N Engl J Med 2002; 346: 1773 1780

4) Kimura T, Morimoto T, Nakagawa $Y$, et al: Very late stent thrombosis and late target lesion revascularization after sirolimus-eluting stent implantation: five-year outcome of the j-Cypher Registry. Circulation 2012; 125: 584-591

5) Tada T, Byrne RA, Simunovic I, et al: Risk of stent thrombosis among bare-metal stents, first-generation drug-eluting stents, and second-generation drug-eluting stents: results from a registry of 18,334 patients. JACC Cardiovasc Interv 2013; 6: 1267 1274

6) Anderson PG, Bajaj RK, Baxley WA, et al: Vascular pathology of balloon-expandable flexible coil stents in humans. J Am Coll Cardiol 1992; 19: 372-381

7) Farb A, Sangiorgi G, Carter AJ, et al: Pathology of acute and chronic coronary stenting in humans. Circulation 1999; 99: 44-52

8) Kimura T, Abe K, Shizuta S, et al: Long-term clinical and angiographic follow-up after coronary stent placement in native coronary arteries. Circulation 2002; 105: 2986-2991

9) Walters DL, Harding SA, Walsh CR, et al: Acute coronary syndrome is a common clinical presentation of in-stent restenosis. Am J Cardiol 2002; 89: 491-494

10) Nayak AK, Kawamura A, Nesto RW, et al: Myocardial infarc- 
tion as a presentation of clinical in-stent restenosis. Circ $\mathrm{J}$ 2006; 70: 1026-1029

11) Chen MS, John JM, Chew DP, et al: Bare metal stent restenosis is not a benign clinical entity. Am Heart J 2006; 151: 12601264

12) Nakazawa G, Otsuka F, Nakano M, et al: The pathology of neoatherosclerosis in human coronary implants bare-metal and drug-eluting stents. J Am Coll Cardiol 2011; 57: 1314-1322

13) Otsuka F, Byrne RA, Yahagi K, et al: Neoatherosclerosis: overview of histopathologic findings and implications for intravascular imaging assessment. Eur Heart J 2015; 36: 2147-2159

14) Ueda $Y$, Nanto $S$, Komamura $K$, et al: Neointimal coverage of stents in human coronary arteries observed by angioscopy. J Am Coll Cardiol 1994; 23: 341-346

15) Oyabu J, Ueda Y, Ogasawara N, et al: Angioscopic evaluation of neointima coverage: sirolimus drug-eluting stent versus bare metal stent. Am Heart J 2006; 152: 1168-1174

16) Higo T, Ueda $Y$, Oyabu J, et al: Atherosclerotic and thrombogenic neointima formed over sirolimus drug-eluting stent: an angioscopic study. JACC Cardiovasc Imaging 2009; 2: 616624

17) Kawakami H, Matsuoka H, Nakamura M, et al: Angioscopic observation three months after sirolimus-eluting stent implantation: Can we stop strong anti-platelet therapy after three months? Jpn Interv Cardiol 2006; 21: 409-416

18) Ueda $Y$, Matsuo K, Nishimoto $Y$, et al: In-Stent Yellow Plaque at 1 Year After Implantation Is Associated With Future Event of Very Late Stent Failure: The DESNOTE Study (Detect the Event of Very late Stent Failure From the Drug-Eluting Stent Not Well Covered by Neointima Determined by Angioscopy). JACC Cardiovasc Interv 2015; 8: 814-821

19) Takano M, Jang IK, Inami $S$, et al: In vivo comparison of optical coherence tomography and angioscopy for the evaluation of coronary plaque characteristics. Am J Cardiol 2008; 101: 471476

20) Ueda $Y$, Ohtani T, Shimizu M, et al: Assessment of plaque vulnerability by angioscopic classification of plaque color. Am Heart J 2004; 148: 333-335

21) Inoue $T$, Shinke $T$, Otake $H$, et al: Neoatherosclerosis and mural thrombus detection after sirolimus-eluting stent implantation. Circ J 2014; 78: 92-100

22) Takano M, Yamamoto M, Inami S, et al: Appearance of lipid-laden intima and neovascularization after implantation of bare-metal stents extended late-phase observation by intracoronary optical coherence tomography. J Am Coll Cardiol 2009; 55: 26-32

23) Kang SJ, Mintz GS, Akasaka T, et al: Optical coherence tomographic analysis of in-stent neoatherosclerosis after drug-eluting stent implantation. Circulation 2011; 123: 2954-2963

24) Yonetsu T, Bouma BE, Kato K, et al: Optical coherence tomography- 15 years in cardiology. Circ J 2013; 77: 1933-1940

25) Tearney GJ, Regar E, Akasaka T, et al: Consensus standards for acquisition, measurement, and reporting of intravascular optical coherence tomography studies: a report from the International Working Group for Intravascular Optical Coherence To- mography Standardization and Validation. J Am Coll Cardiol 2012; 59: 1058-1072

26) Jang IK, Tearney GJ, MacNeill B, et al: In vivo characterization of coronary atherosclerotic plaque by use of optical coherence tomography. Circulation 2005; 111: 1551-1555

27) Tearney GJ, Yabushita H, Houser SL, et al: Quantification of macrophage content in atherosclerotic plaques by optical coherence tomography. Circulation 2003; 107: 113-119

28) Gonzalo N, Serruys PW, Okamura T, et al: Optical coherence tomography patterns of stent restenosis. Am Heart J 2009; 158: 284-293

29) Yonetsu T, Kato K, Kim SJ, et al: Predictors for neoatherosclerosis: a retrospective observational study from the optical coherence tomography registry. Circ Cardiovasc Imaging 2012; 5: 660-666

30) Yonetsu T, Kim JS, Kato K, et al: Comparison of incidence and time course of neoatherosclerosis between bare metal stents and drug-eluting stents using optical coherence tomography. Am J Cardiol 2012; 110: 933-939

31) Lee SY, Hur SH, Lee SG, et al: Optical coherence tomographic observation of in-stent neoatherosclerosis in lesions with more than $50 \%$ neointimal area stenosis after second-generation drug-eluting stent implantation. Circ Cardiovasc Interv 2015; 8: $\mathrm{e} 001878$

32) van Soest G, Regar E, Goderie TP, et al: Pitfalls in plaque characterization by OCT: image artifacts in native coronary arteries. JACC Cardiovasc Imaging 2011; 4: 810-813

33) Nakano M, Vorpahl M, Otsuka F, et al: Ex vivo assessment of vascular response to coronary stents by optical frequency domain imaging. JACC Cardiovasc Imaging 2012; 5: 71-82

34) Hou J, Qi H, Zhang M, et al: Development of lipid-rich plaque inside bare metal stent: possible mechanism of late stent thrombosis? An optical coherence tomography study. Heart 2010; 96: 1187-1190

35) Habara M, Terashima M, Nasu K, et al: Difference of tissue characteristics between early and very late restenosis lesions after bare-metal stent implantation: an optical coherence tomography study. Circ Cardiovasc Interv 2011; 4: 232-238

36) Habara M, Terashima M, Nasu K, et al: Morphological differences of tissue characteristics between early, late, and very late restenosis lesions after first generation drug-eluting stent implantation: an optical coherence tomography study. Eur Heart J Cardiovasc Imaging 2013; 14: 276-284

37) Otsuka F, Vorpahl M, Nakano M, et al: Pathology of second-generation everolimus-eluting stents versus first-generation sirolimus- and paclitaxel-eluting stents in humans. Circulation 2014; 129: 211-223

38) Palmerini T, Biondi-Zoccai G, Stone GW: Stent selection to minimize the risk of stent thrombosis. Curr Opin Cardiol 2014; 29: 578-585

39) Kubo T, Akasaka T, Kozuma K, et al: Comparison of neointimal coverage between everolimus-eluting stents and sirolimus-eluting stents: an optical coherence tomography substudy of the RESET (Randomized Evaluation of Sirolimus-eluting versus Everolimus-eluting stent Trial). EuroIntervention 2015; 11: 
564-571

40) Finn AV, Joner M, Nakazawa G, et al: Pathological correlates of late drug-eluting stent thrombosis: strut coverage as a marker of endothelialization. Circulation 2007; 115: 24352441

41) Guagliumi G, Sirbu V, Musumeci G, et al: Examination of the in vivo mechanisms of late drug-eluting stent thrombosis: findings from optical coherence tomography and intravascular ultrasound imaging. JACC Cardiovasc Interv 2012; 5: 12-20

42) Amabile N, Souteyrand G, Ghostine S, et al: Very late stent thrombosis related to incomplete neointimal coverage or neoatherosclerotic plaque rupture identified by optical coherence tomography imaging. Eur Heart J Cardiovasc Imaging 2014; 15: 24-31

43) Souteyrand G, Amabile N, Mangin L, et al: Mechanisms of stent thrombosis analysed by optical coherence tomography: insights from the national PESTO French registry. Eur Heart J 2016; 37: 1208-1216

44) Taniwaki M, Radu MD, Zaugg S, et al: Mechanisms of Very Late Drug-Eluting Stent Thrombosis Assessed by Optical Coherence Tomography. Circulation 2016; 133: 650-660

45) Taniwaki M, Windecker S, Zaugg S, et al: The association between in-stent neoatherosclerosis and native coronary artery disease progression: a long-term angiographic and optical coherence tomography cohort study. Eur Heart J 2015; 36: $2167-$ 2176

46) Kuroda M, Otake H, Shinke T, et al: The impact of in-stent neoatherosclerosis on long-term clinical outcomes: an observational study from the Kobe University Hospital optical coherence tomography registry. EuroIntervention 2016; 12: e1366-e1374 